PHYSICAL REVIEW D 84, 103525 (2011)

\title{
Relevance of sharp gamma-ray features for indirect dark matter searches
}

\author{
Torsten Bringmann* and Francesca Calore ${ }^{\dagger}$ \\ II. Institute for Theoretical Physics, University of Hamburg, Luruper Chaussee 149, DE-22761 Hamburg, Germany \\ Gilles Vertongen \\ Deutsches Elektronen-Synchrotron (DESY), Notkestrasse 85, 22603 Hamburg, Germany \\ Christoph Weniger ${ }^{\S}$ \\ Max-Planck-Institut für Physik, Föhringer Ring 6, 80805 Munich, Germany
}

(Received 23 June 2011; published 22 November 2011)

\begin{abstract}
Gamma rays from the annihilation of dark matter particles in the Galactic halo provide a particularly promising means of indirectly detecting dark matter. Here, we demonstrate that pronounced spectral features at energies near the dark matter particles' mass, which are a generic prediction for most models, can significantly improve the sensitivity of gamma-ray telescopes to dark matter signals. We derive projected limits on such features (including the traditionally looked-for line signals) and show that they can be much more efficient in constraining the nature of dark matter than the model-independent broad spectral features expected at lower energies.
\end{abstract}

DOI: 10.1103/PhysRevD.84.103525

PACS numbers: 95.35.+d, 29.40.Ka, 95.55.Ka

\section{INTRODUCTION}

Indirect dark matter (DM) searches aim at seeing an excess in cosmic rays from the annihilation or decay of DM in the Galactic halo [1]. Gamma rays play a pronounced role in this respect because they are produced rather copiously and directly trace their sources as they propagate essentially unperturbed through the galaxy. Powerful currently operating telescopes like Fermi LAT [2], HESS [3], MAGIC [4] or VERITAS [5] now start to constrain viable DM models and next generation instruments like the planned CTA [6] will be able to dig quite a bit into the underlying parameter space of particle physics models, in a way complementary to both direct DM detection and searches at the CERN LHC [7].

Very often, indirect searches focus on secondary photons from the fragmentation of annihilation products, mostly via $\pi^{0} \rightarrow \gamma \gamma$. The resulting spectrum is rather model-independent and would manifest itself as a broad bumplike excess over the expected background at energies considerably lower than the DM mass $m_{\chi}$. Convincingly claiming a DM detection based on the observation of such a featureless signal will generically be difficult.

In many models, however, pronounced spectral features are expected at the kinematical endpoint $E_{\gamma}=m_{\chi}$ and include monochromatic gamma-ray lines [8], sharp steps or cutoffs $[9,10]$ as well as pronounced bumps [11]. The type and strength of these features are intricately linked to the particle nature of DM; a detection would thus not only allow a convincing discrimination from astrophysical

\footnotetext{
*torsten.bringmann@desy.de

†francesca.calore@desy.de

*gilles.vertongen@desy.de

§weniger@mppmu.mpg.de
}

backgrounds but also to determine important DM model parameters (in particular, but not necessarily limited to, the value of $m_{\chi}$ ). So far, only line-signals have explicitly been searched for [12] - despite the fact that they are loopsuppressed and thus generically subdominant compared to other spectral signatures [11].

Here, we present a general method to search for such features and show that these, indeed, help significantly to discriminate DM signals from astrophysical backgrounds. This allows us to derive very competitive (projected) limits on both the annihilation rate and nature of DM, which we believe will be very useful for DM searches.

\section{METHOD}

The defining aspect of the above-mentioned spectral features in the DM-induced gamma-ray emission is an abrupt change of the flux as a function of energy; in the extreme cases of gamma-ray lines or cutoffs, e.g., the corresponding energy range would simply be given by the energy resolution $\Delta E / E$ of the instrument. The basic idea that we will adopt here, following traditional gammaray line searches [12], is therefore to concentrate the search for spectral features on a small sliding energy window $\left[E_{0}, E_{1}\right]$, with $E_{0}<m_{\chi}<E_{1}$ and $\varepsilon \equiv E_{1} / E_{0} \sim \mathcal{O}(1-10)$. An important advantage of using small values for $\varepsilon$ is that gamma-ray fluxes with astrophysical origin can often be very well described by a simple power-law. In that case, a corresponding fit to the data allows an effective determination of the background at the statistical limit, greatly reducing uncertainties related to astrophysical sources.

For deriving constraints on spectral features within the sliding energy window, we will use a binned profile likelihood method [13]. To this end, we split $\left[E_{0}, E_{1}\right]$ in many 
energy bins $\Delta E_{i}$ and define a likelihood function $L(\mu \mid \mathbf{c})=$ $\Pi_{i} P_{\mu_{i}}\left(c_{i}\right)$, where $\mu_{i}\left(c_{i}\right)$ denotes the expected (observed) count number in bin $i$ and $P_{\mu}$ is the Poisson probability distribution with mean $\mu$. Introducing the background normalization $\beta$, its spectral slope $\gamma$ and the normalization of the DM signal $\alpha$, we have

$\frac{\mu_{i}}{t_{\mathrm{obs}}}=\int_{\Delta E_{i}} d E \int d E^{\prime} \mathcal{D}_{E, E^{\prime}} A_{\mathrm{eff}}\left(E^{\prime}\right)\left[\alpha \frac{d N_{\chi}}{d E^{\prime}}+\beta E^{\prime-\gamma}\right]$,

where $t_{\mathrm{obs}}$ is the time of observation, $A_{\text {eff }}$ the effective area and $\mathcal{D}_{E, E^{\prime}}$ the energy dispersion of the instrument (in the following taken to be Gaussian). Maximizing $L(\mu \mid \mathbf{c})$ for a given data set $\mathbf{c}$ results in best-fit values of the model parameters $\alpha, \beta$ and $\gamma$ within the considered window $\left[E_{0}, E_{1}\right]$. Upper limits on the signal strength at the $95.5 \%$ C.L. can then be derived by increasing $\alpha$ from its best-fit value until $-2 \log L$ (maximized with respect to $\beta$ and $\gamma$ ) has changed by 4 . On the other hand, a detection at the $5 \sigma$ level (neglecting trial factors) could be claimed if the best-fit $-2 \log L$ values for background-only and background-plus-signal fits differ by at least 25 .

Our main assumption here is that the astrophysical background locally takes the form of a power law. Obviously, this approximation can break down in case of large window sizes $\varepsilon$, depending on the collected statistics and, to first order, on the intrinsic curvature of the background flux $\kappa \equiv d^{2} \log \left(d J_{\mathrm{BG}} / d E\right) /(d \log E)^{2}$ : a change in the spectral index by $\Delta \gamma$, e.g., implies roughly $|\kappa| \sim$ $\Delta \gamma^{2} / 4$ at the transition point; $\kappa$ could, however, also be affected by systematic uncertainties in $A_{\text {eff }}$. We will derive constraints on the maximally allowed window size $\varepsilon_{\max }$ by requiring that these effects do not significantly alter the resulting DM limits.

\section{CHOICE OF TARGET AND INSTRUMENT SPECIFICATIONS}

For concreteness, we will in the following focus on observations of the Galactic center region with Imaging Atmospheric Cherenkov Telescopes (IACTs). We consider the benchmark scenarios summarized in Table I, which roughly correspond to the telescope characteristics of the currently operating HESS [3], the future CTA [6] and-as the most optimistic choice for indirect DM searches-the proposed Dark Matter Array (DMA) [7]. We implement the energy dependence of the effective

TABLE I. IACT benchmark models that, from top to bottom, roughly correspond to the HESS [3], the future CTA [6] and the proposed DMA [7] telescope characteristics.

\begin{tabular}{lcccr}
\hline \hline & $A_{\mathrm{eff}}(1 \mathrm{TeV})$ & $\Delta E / E(1 \mathrm{TeV})$ & $\epsilon_{p}$ & \multicolumn{1}{c}{$t_{\mathrm{obs}}$} \\
\hline IACT1 & $0.18 \mathrm{~km}^{2}$ & $15 \%$ & $10^{-1}$ & $50 \mathrm{~h}$ \\
IACT2 & $2.3 \mathrm{~km}^{2}$ & $9 \%$ & $10^{-2}$ & $100 \mathrm{~h}$ \\
IACT3 & $23 \mathrm{~km}^{2}$ & $7 \%$ & $10^{-3}$ & $5000 \mathrm{~h}$ \\
\hline \hline
\end{tabular}

area $A_{\text {eff }}$ as given in Ref. [3] (Ref. [14]) for HESS (CTA) and take $A_{\mathrm{eff}}^{\mathrm{DMA}}=10 \cdot A_{\mathrm{eff}}^{\mathrm{CTA}}$. The proton, gamma-ray and electron efficiencies $\epsilon_{p, \gamma, e^{-}}$in all three scenarios as well as the energy resolution $\Delta E / E$ in case of HESS and DMA are taken to be energy independent; for CTA we adopt results from Ref. [6]. We will use $\epsilon_{\gamma}=\epsilon_{e^{-}}=0.8$ throughout and assume that the proposed DMA can reject protons with efficiencies $\epsilon_{p} \approx 10^{-3}$.

For the background, we take into account cosmic-ray fluxes of electrons [15] and protons [16,17], the diffuse gamma-ray flux [18] and the source HESS J1745-290 [19] at (or very close to) the Galactic center. A summary and more detailed description can be found in the Appendix; there, we also discuss which choice of target region $\Delta \Omega$ optimizes the signal-to-noise ratio $\mathcal{S} / \mathcal{N}$ (see also Ref. [20]). For the Einasto and NFW DM profiles, with parameters as in Ref. [21], we will adopt a relatively small region $\Delta \Omega=2^{\circ} \times 2^{\circ}$ around the Galactic center; larger regions would weaken the signal-to-background ratio, $\mathcal{S} / \mathcal{B}$. In case of a strong pointsource like enhancement of the DM signal from the Galactic center (e.g. through the effect of the supermassive black hole $[22,23]$ or adiabatic compression [24-26]), it is favorable to focus on even much smaller target regions. As an example, we consider the case of an adiabatically compressed (AC) profile $[25,26]$ for which we choose a target region of $\Delta \Omega=$ $0.2^{\circ} \times 0.2^{\circ}$.

Let us now derive values for the tolerable window size $\varepsilon_{\max }$ in the presence of maximal background curvatures $\kappa_{\max }$. Using mock data sets with $\kappa= \pm \kappa_{\max }$, we compare average DM limits (on the DM models introduced below) obtained when using the power-law ansatz for the background with the limits obtained when incorporating a (fixed) curvature $\kappa$ in the background fit. In Fig. 1 we display as function of the sliding energy window position

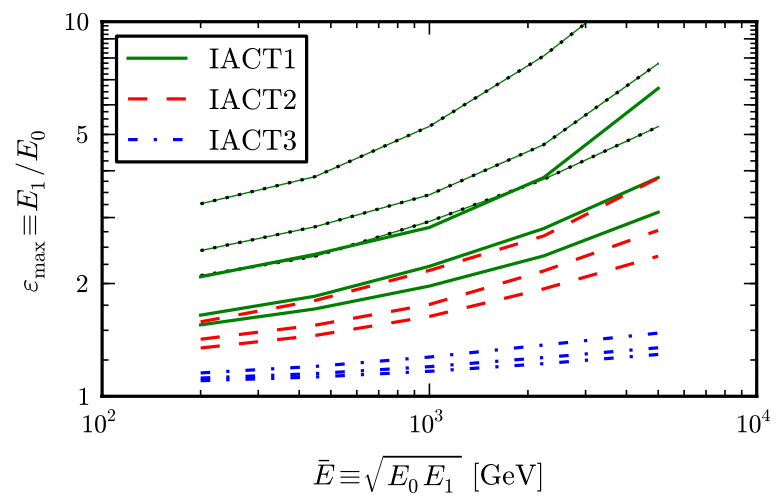

FIG. 1 (color online). Maximal sliding energy window size $\varepsilon_{\max }$ as function of the window position $\bar{E}$. We show for different intrinsic background curvatures $\kappa_{\max }=0.1,0.2,0.3$ (top to bottom) the window sizes above which DM limits are affected by more than $50 \%$. The dotted lines show for IACT1 $\varepsilon_{\max }$ for which a power-law ansatz would still give a good fit to the background. 
$\bar{E} \equiv \sqrt{E_{0} E_{1}}$, and for different curvatures $\kappa_{\max }$, the values of $\varepsilon_{\max }$ above which the DM limits are affected by more than $50 \%$. For comparison, the dotted lines show for IACT1 the values for $\varepsilon_{\max }$ below which the power-law fit still appears to be in good agreement with the curved background (using as criterion that for at least $80 \%$ of the mock data sets the $p$-value of the power-law fit is larger than 0.05): obviously, a good quality of the power-law fit alone does not automatically exclude sizeable effects on the DM limits. Therefore, a priori assumptions on $\kappa_{\max }$ are indispensable; in our case, we employ $|\kappa| \leq \kappa_{\max } \approx 0.2$-which we checked to be satisfied for the background we adopt here-to determine optimal logarithmic window sizes for IACT 1 and IACT 2 according to Fig. 1 (for IACT3, see below).

\section{DARK MATTER SPECTRAL SIGNATURES}

The DM signal flux from a sky region $\Delta \Omega$ is given by

$$
\begin{aligned}
\frac{d J_{\chi}}{d E} & \equiv \alpha \frac{d N_{\chi}}{d E} \\
& =\frac{\langle\sigma v\rangle}{8 \pi m_{\chi}^{2}} \int_{\Delta \Omega} d \Omega \int_{\text {l.o.s. }} d s \rho_{\chi}(r(s, \Omega))^{2} \frac{d N_{\chi}}{d E},
\end{aligned}
$$

where $\langle\sigma v\rangle$ is the annihilation rate, $d N_{\chi} / d E$ the differential number of photons per annihilation, $\rho_{\chi}(r)$ the Galactic DM profile and $s$ runs over the line-of-sight. For any photon spectrum, and a given value of the dark matter particles' mass, we can now derive limits on $\alpha$ (aka $\langle\sigma v\rangle)$ by scanning over all possible values of $m_{\chi}$ and applying the method described in detail in Sec. II. As a technical remark, we found that the best limits are actually obtained by choosing the center of the sliding energy window to lie slightly off-set from the kinematic endpoint $E=m_{\chi}$ of DM spectra at or slightly below which we expect to see the features we are looking for. For the instrument specifications and background model that we adopted here, in particular, the optimal choice turned out to be $\bar{E}=\varepsilon^{-0.25} m_{\chi}$ (not for line signals, however, for which we take $\bar{E}=m_{\chi}$ ).

In the following, we will discuss three types of typical endpoint features that arise from radiative corrections to the tree-level annihilation process. The most striking spectral signature, in terms of a possible discrimination from a power-law background, is a gamma-ray line at $E_{\gamma}=m_{\chi}\left(E_{\gamma}=m_{\chi}\left[1-m_{Z / H}^{2} / 4 m_{\chi}^{2}\right]\right)$, which would result from the direct annihilation of DM into $\gamma \gamma(Z \gamma$ or $H \gamma)$ [8]. Generically, for thermal cross sections of DM in the form of weakly interacting massive particles (WIMPs), the annihilation rate is expected to be of the order of $\langle\sigma v\rangle_{\text {line }} \sim$ $\alpha_{\mathrm{em}}^{2} \times\langle\sigma v\rangle_{\text {tree }} \sim 10^{-30} \mathrm{~cm}^{3} \mathrm{~s}^{-1}$, but in some cases much stronger line signals are possible [27-29].

As an example for a steplike feature we use the gammaray spectrum [10] expected from annihilating KaluzaKlein $(K K) D M$ in models of universal extra dimensions [30]. In the minimal version of these models, the DM particle is the $B^{(1)}$, i.e. the first KK excitation of the weak hypercharge gauge boson, and the correct relic density is obtained for $m_{B^{(1)}} \sim 1.3 \mathrm{TeV}$ [31]. Its total gamma-ray annihilation spectrum $d N / d x$ (with $x \equiv E / m_{\chi}$ ) at high energies is dominated by final state radiation (FSR) off lepton final states and turns out to be essentially independent of $m_{B^{(1)}}$ and other model parameters.

Pronounced bumplike features at $E \simeq m_{\chi}$ may arise from internal bremsstrahlung (IB) in the annihilation of neutralino DM [11]. While these spectra are in general highly model-dependent, we follow here a simplified approach by defining two spectral templates $d N / d x$ (which we take to be independent of $m_{\chi}$ ) by referring to neutralino benchmark models introduced in Ref. [11]. Here, BM3 is a typical example for a neutralino in the stau coannihilation region, where photon emission from virtual sleptons greatly enhances $d N / d x$; $\mathrm{BM} 4$ refers to a situation in which IB from $W^{ \pm}$final states dominates. We note that the Sommerfeld effect could strongly enhance these features, in particular in the case of BM4, in the same way as pointed out in Ref. [27] for line signals.

In Table II, we shortly summarize the properties of the DM benchmark models described above, including for completeness the actual DM mass and total annihilation rate needed to obtain the observed relic density for thermally produced DM. Note, however, that we essentially treat these values as free parameters in our analysis and that we are rather interested in the spectral shape of the annihilation signal, represented by $d N / d x$; in Fig. 2 we show these spectra for a direct comparison.

TABLE II. DM benchmark models used in our analysis as examples for the typical spectral endpoint features to be expected in WIMP annihilations. For these particular models, we also state the annihilation channel that is most important in this context, as well as mass and total annihilation rate for thermally produced DM. See text for further details about the DM models and Fig. 2 for the corresponding photon spectra.

\begin{tabular}{lccccc}
\hline \hline & DM particle & $m_{\chi}^{\text {th }}[\mathrm{TeV}]$ & $\langle\sigma v\rangle$ th $\left[\mathrm{cm}^{3} \mathrm{~s}^{-1}\right]$ & relevant channel & spectral feature \\
\hline$\gamma \gamma$ & any WIMP & $\mathcal{O}(0.1-10)$ & $\mathcal{O}\left(10^{-30}\right)$ & $\gamma \gamma$ & line \\
KK & $B^{(1)}$ & 1.3 & $1 \times 10^{-26}$ & $\ell^{+} \ell^{-} \gamma$ & FSR step \\
BM3 & neutralino & 0.23 & $9 \times 10^{-29}$ & $\ell^{+} \ell^{-} \gamma$ & IB bump \\
BM4 & neutralino & 1.9 & $3 \times 10^{-27}$ & $W^{+} W^{-} \gamma$ & IB bump \\
\hline \hline
\end{tabular}




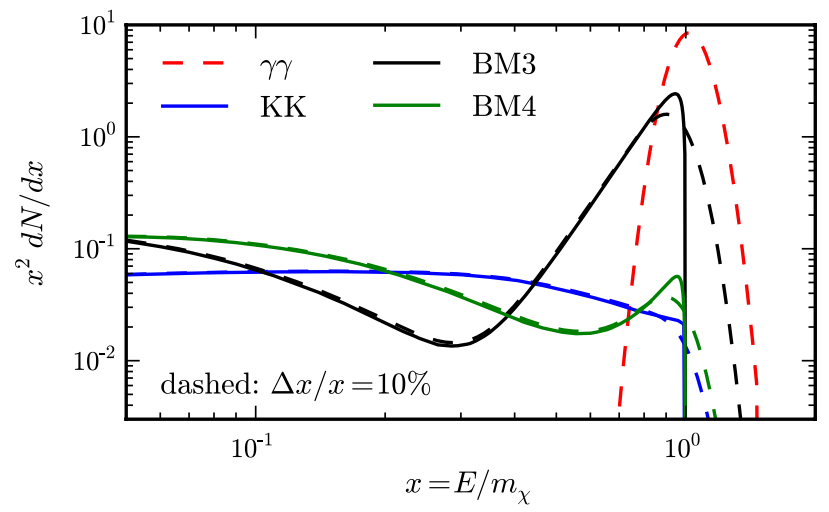

FIG. 2 (color online). Photon spectra for the DM benchmark models of Table II. Dashed lines show the same spectra, smeared with a Gaussian of width $\Delta x / x=0.1$ to give a rough indication of how well a detector with such an energy resolution would in principle be able to discriminate these models from astrophysical (power-law) backgrounds, as well as from each other.

\section{LIMITS AND DISCUSSION}

In Fig. 3 we show our results for the expected $2 \sigma$ upper limits (thick lines) on the above DM models as well as the variance of these limits among the 300 mock data sets that we created for this analysis. We find that, in particular, IB features in the spectrum (right panel) have the potential to constrain the annihilation rate at least down to values typically expected for thermal production, $\langle\sigma v\rangle \sim 3 \cdot 10^{-26} \mathrm{~cm}^{3} \mathrm{~s}^{-1}$, already for modest assumptions about the DM distribution (we verified that NFW and
Einasto profiles give similar results). This is very competitive compared to the best current limits from ACTs that only rely on secondary photons [32] — though we would like to stress that these limits provide rather complementary information on the DM nature and can thus usually not easily be compared.

For the case of not too strongly pronounced endpoint features (like line signals in most models or the step for Kaluza-Klein DM), secondary photons will usually be more powerful in constraining the total annihilation rate $\langle\sigma v\rangle$; from the point of view of indirect DM searches, however, the detection of the kinematic cutoff will be much more interesting than the detection of secondary photons since it allows to draw firmer conclusions about the DM origin of the signal and even to determine important parameters like $m_{\chi}$. For models with very large IB contributions like BM3, on the other hand, we find that our method provides even stronger limits on $\langle\sigma v\rangle$ than what was obtained by the HESS analysis of the Galactic center region assuming annihilation into $\bar{b} b$ [32].

In case of an adiabatically compressed profile our limits could improve by 2 orders of magnitude, as demonstrated for gamma-ray lines in the left panel; under such conditions, one could even hope to constrain models with very small annihilation rates like BM3 (recall that the annihilation rate for BM4 is affected anyway by the Sommerfeld enhancement [27] and thus likely considerably larger than what is shown in Fig. 3). As shown in the central panel of Fig. 3, the future CTA should be able to place limits about 1 order of magnitude stronger than currently possible, and

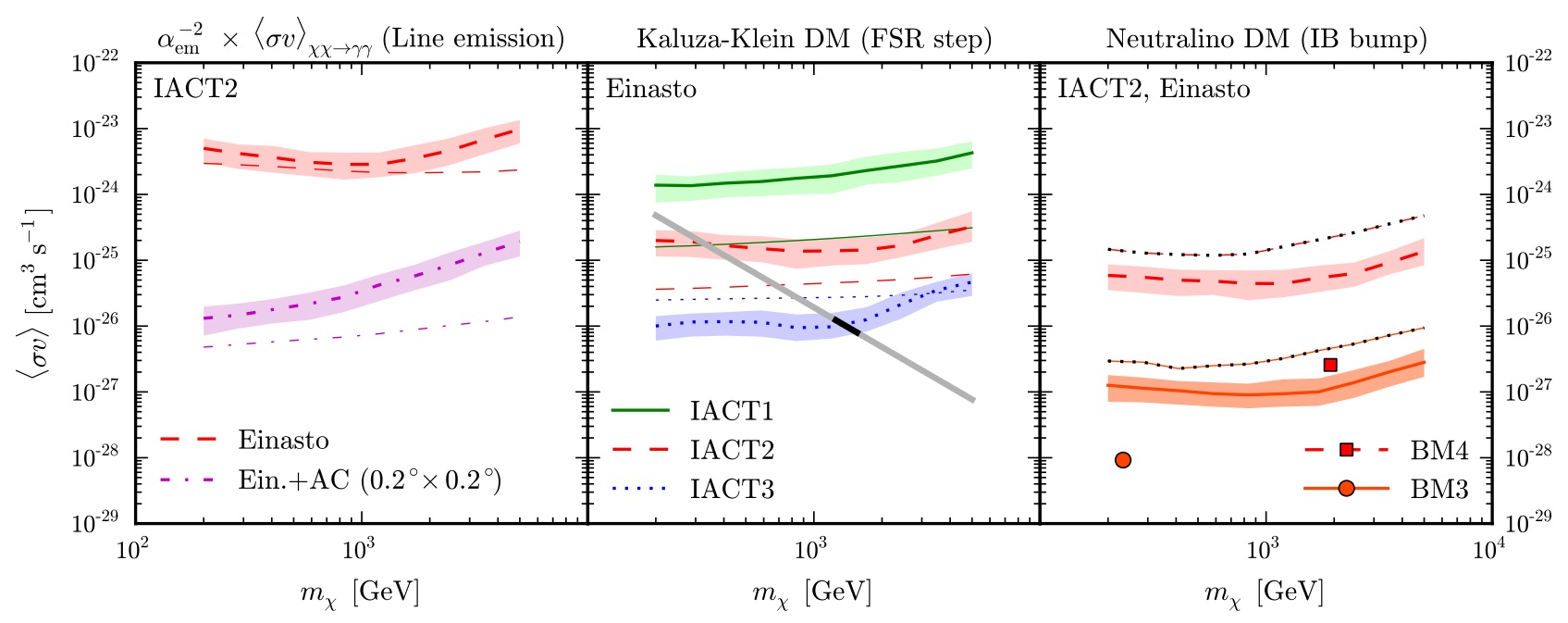

FIG. 3 (color online). Thick lines: Expected $2 \sigma$ upper limits on $\langle\sigma v\rangle$ for selected DM models, DM profiles and observational scenarios; bands indicate the variance of these limits. Thin lines: Spectral feature of DM signal has $\mathcal{S} / \mathcal{B} \approx 1 \%$ (after convolution with energy dispersion). The left panel shows limits on gamma-ray lines, rescaled by a loop-factor of $\alpha_{\mathrm{em}}^{-2}$ for better comparison. In the central panel, the gray band indicates the expected $\langle\sigma v\rangle$ for KK DM, the black part being compatible with the observed relic density. In the right panel, we indicate the adopted neutralino benchmark points, and the dotted lines show the projected $5 \sigma$ sensitivity. 
the proposed DMA could further improve these by another factor of $10 .^{1}$

When probing a specific DM model, the corresponding $\mathcal{S} / \mathcal{B}$ is a good measure for the level on which spectral artifacts in the energy reconstruction of the instrument must be understood. As can be inferred from Fig. 3 (thin lines), most of our derived limits correspond to moderate $\mathcal{S} / \mathcal{B}$ values of at least a few percent (except for IACT3), which should be well in reach of current instruments.

Limits on gamma-ray lines as shown in Fig. 3 are usually derived neglecting any secondary gamma-ray component from DM annihilation [12]; this approximation, however, breaks down for very small branching ratio into lines since part of the secondary component will leak into the sliding energy window. Assuming a dominant annihilation into $b \bar{b}$ final states, we find that for branching ratios into gamma-ray lines smaller than $\mathcal{O}\left(10^{-4}\right)$, the presence of the secondary flux begins to alter the derived gamma-ray line limits significantly. This renders a naive application of standard line-search results on DM models with generic $\mathcal{O}\left(\alpha_{\mathrm{em}}^{2}\right)$ branching ratios into gamma-ray lines questionable [33].

The dotted lines in the right panel of Fig. 3 show the projected sensitivity to see a $5 \sigma$ signal in the IACT2 scenario (neglecting systematics and trial factors). Such an observation should of course be cross-checked by the nonobservation of the same signature in control regions without large DM-induced fluxes. A more detailed analysis for detectional prospects is beyond the scope of the present work and left for a subsequent publication [33].

\section{CONCLUSIONS}

Gamma rays from DM annihilation often exhibit pronounced spectral features near photon energies close to the DM particles' mass. Here, we have shown that methods from gamma-ray line searches, which greatly reduce the uncertainties related to astrophysical background fluxes, can successfully be extended to look for such spectral features; this provides a probe of the DM nature that is complementary to DM searches relying only on the rather model-independent spectrum from secondary photons.

While these kind of features may generically be considered even more relevant for the detection of DM signals, because they would provide rather unambiguous evidence for the DM nature of the signal as well as allow to determine important parameters like the DM mass, we have demonstrated here that including the spectral information may even significantly improve limits on DM signals; steps

\footnotetext{
${ }^{1}$ Note that for DMA, as can be seen from Fig. 1, the statistics actually become so good that a spectrum with $\kappa \sim \mathcal{O}(0.1)$ curvature starts to deviate significantly from a power-law background already for rather small sliding energy windows. In order to obtain reasonable limits, we therefore included $\kappa \neq 0$ as a free parameter in the fit to allow for energy windows somewhat larger than shown in Fig. 1.
}

or bumplike IB features can, in fact, be much more important in this respect than lines.

We stress that while we have considered constraints for IACT observations of the Galactic center region, the presented method is much more general and can be applied to both other targets and other instruments; we thus expect it to be useful for a wide range of applications in indirect DM searches. An obvious extension of the approach presented here, finally, is to apply it to the detection rather than exclusion of DM signals, as well as to the discrimination of models [33].

\section{ACKNOWLEDGMENTS}

We thank U. Almeida, D. Borla Tridon and H. Zechlin for useful discussions, and M. Kakizaki for confirming that the gamma-ray spectrum of KK DM computed in Ref. [10] remains essentially unaffected by changing the DM mass such as to be compatible with the most recent relic density calculations [31]. T. B. and F. C. acknowledge support from the German Research Foundation (DFG) through Emmy Noether grant BR 3954/1-1.

\section{APPENDIX: DARK MATTER SEARCHES IN THE GALACTIC CENTER REGION}

Imaging Atmospheric Cherenkov Telescopes (IACTs) detect gamma rays by measuring the dim Cherenkov light produced by electromagnetic showers through the atmosphere. Very similar showers are induced by cosmic-ray electrons, which hence constitute a practically irreducible background. Proton-induced hadronic showers, on the other hand, differ in profile and energy density and can currently be rejected with efficiencies $\epsilon_{p} \sim \mathcal{O}\left(10^{-2}-10^{-1}\right)$. Because of their large intrinsic fluxes, charged cosmic rays typically form the major background of IACT observations. For the flux of cosmic-ray electrons, we take $d J_{e^{-}} / d E d \Omega=$ $1.17 \times 10^{-11}(E / \mathrm{TeV})^{-3.9}\left(\mathrm{GeV} \mathrm{cm}^{2} \mathrm{~s} \mathrm{sr}\right)^{-1}$ above $1 \mathrm{TeV}$, which hardens below $1 \mathrm{TeV}$ to a spectral index of -3.0 [15] (with a transition between the two fluxes that we assume to be proportional to their generalized mean with exponent -2 ). For the proton flux we take $d J_{p} / d E d \Omega=$ $8.73 \times 10^{-9}(E / \mathrm{TeV})^{-2.71}\left(\mathrm{GeV} \mathrm{cm}^{2} \mathrm{~s} \mathrm{sr}\right)^{-1}$ [16], which we shift to lower energies by a factor of 3 to take into account the reduced Cherenkov light output of hadronic showers, $E_{p}^{\text {recon }} \approx E_{p}^{\text {true }} / 3$ (see e.g. Ref. [17]).

For observations of the Galactic Center region (GC), we take as further background into account the HESS source J1745-290 [19], with $d J_{\mathrm{HESS}} / d E=$ $2.3 \times 10^{-15}(E / \mathrm{TeV})^{-2.25}\left(\mathrm{GeV} \mathrm{cm}^{2} \mathrm{~s}\right)^{-1}$. The diffuse photon emission measured by HESS in a $-0.8^{\circ} \leq \ell \leq 0.8^{\circ}$ and $|b| \leq 0.3^{\circ}$ region around the $\mathrm{GC}$ is given by $d J_{\text {diff }} / d E=5.1 \times 10^{-15}(E / \mathrm{TeV})^{-2.29}\left(\mathrm{GeV} \mathrm{cm}^{2} \mathrm{~s}\right)^{-1} \quad[18]$. Unknown diffuse emission from outside this region will conservatively be accounted for by upscaling this flux by a factor of 2 within our $2^{\circ} \times 2^{\circ}$ target region. We summarize all these background contributions in Fig. 4. 


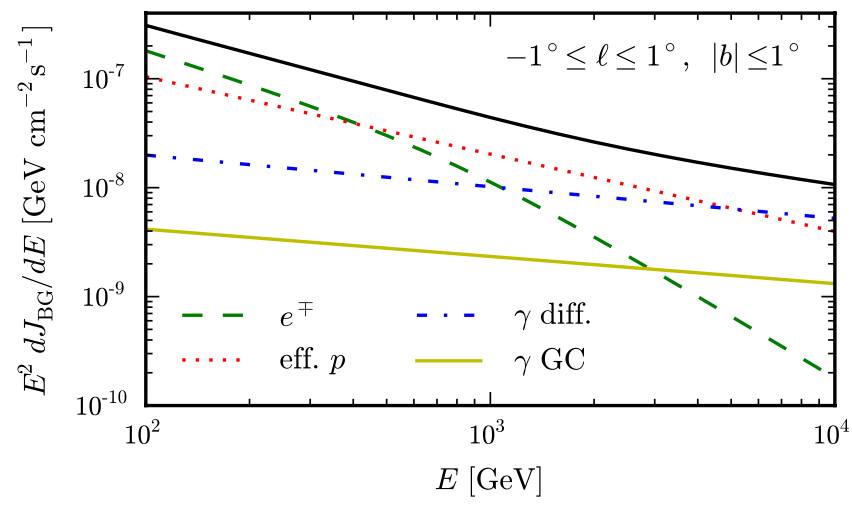

FIG. 4 (color online). Summary of adopted background fluxes in a $2^{\circ} \times 2^{\circ}$ region around the Galactic center, with an effective proton flux as it enters the IACT2 scenario. The black solid line shows the sum of all background fluxes.

The statistical significance of a spectral feature depends on the signal-to-noise ratio $\mathcal{S} / \mathcal{N}(\mathcal{N} \simeq \sqrt{\mathcal{B}+\mathcal{S}})$ inside the target region. The number of expected background events $\mathcal{B}$ within a target region $\Delta \Omega$ and energy range $\Delta E$ is calculated analogously to Eq. (1) by replacing the model flux by the sum of the above background fluxes after integrating over $\Delta \Omega$. In the same way, the number of signal events $\mathcal{S}$ follows from the DM annihilation flux as given in Eq. (2).

In Fig. 5, we show the $\mathcal{S} / \mathcal{N}$ (thick lines) of spectral features for a circular region around the GC with radius $\theta$. We compare results for the standard Einasto and NFW DM profiles with parameters as in Ref. [21] (i.e. $r_{s}^{\mathrm{NFW}}=21 \mathrm{kpc}$, $r_{s}^{\text {Ein }}=20 \mathrm{kpc}, \alpha=0.17$ and $\rho_{\chi}=0.4 \mathrm{GeV} \mathrm{cm}^{-3}$ at Sun's position $R_{\odot}=8.5 \mathrm{kpc}$ ). As can be seen from the figure radii of a few degree are required in order to maximize $\mathcal{S} / \mathcal{N}$ for these profiles (see also Ref. [20]).

For the optimal choice of $\Delta \Omega$ one should also consider the signal-to-background ratio $\mathcal{S} / \mathcal{B}$ which is shown in Fig. 5 for comparison (thin lines). $\mathcal{S} / \mathcal{B}$ is related to the importance of systematic instrumental effects for the statistical analysis, i.e. it gives an indication of how well artifacts and uncertainties in the reconstructed energy spectrum of the instrument must be understood. In most of our analysis, we use a relatively small $\Delta \Omega=2^{\circ} \times 2^{\circ}$ region around the GC. As can be seen in Fig. 5, although a

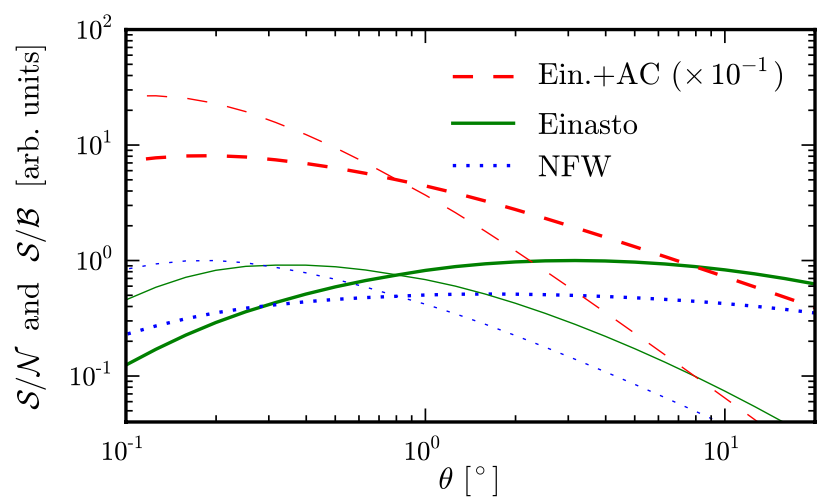

FIG. 5 (color online). $\mathcal{S} / \mathcal{N}$ (thick lines) and $\mathcal{S} / \mathcal{B}$ (thin lines) of DM signal inside a circular region around the GC with radius $\theta$, for different DM halo profiles. For the Einasto profile, we also show the effect of adiabatic compression (AC). We assume $\mathcal{S} \ll$ $\mathcal{B}$ and an energy threshold of $200 \mathrm{GeV}$, but for threshold energies $100 \mathrm{GeV}-5 \mathrm{TeV}$ we find similar results.

larger region could improve $\mathcal{S} / \mathcal{N}$, it also would imply a significantly reduced $\mathcal{S} / \mathcal{B}$.

In some cases, e.g. through the effect of the supermassive black hole [22] or adiabatic compression [24-26], the DM annihilation can be boosted in a region concentrated around the GC, leading to a qualitative change in the behavior of $\mathcal{S} / \mathcal{N}$ with respect to the above unboosted DM profiles. The effect of adiabatic compression is illustrated in Fig. 5 in the case of the Einasto profile $($ Ein + AC), where we exemplarily adopt the adiabatic contraction model of Gnedin et al. [25] together with the best-fit parameters inferred from the hydrodynamical simulation S1 of Gustafsson et al. [26]. In this case, the profile inner slope steepens to $\rho \sim r^{-1.12}$. For such an enhancement, DM self-annihilations start to play a role and constrain the halo density to be at most $\rho_{\max } \sim$ $m_{\chi} /\langle\sigma v\rangle \tau_{\text {gal }}$ [23]; however, the cutoff radii obtained, $r \sim 10^{-9} \mathrm{kpc}$, are so small that this effect does not influence our results. As can be seen from Fig. 5, in the adopted boosted scenario it is preferable to consider much smaller target regions than in case of the above unboosted profiles; hence, we will use a $0.2^{\circ} \times 0.2^{\circ}$ region around the $\mathrm{GC}$ when calculating limits in presence of adiabatic compression.
[1] For a review on particle DM see, e.g., G. Bertone, D. Hooper, and J. Silk, Phys. Rep. 405, 279 (2005).

[2] W. B. Atwood et al. (LAT Collaboration), Astrophys. J. 697, 1071 (2009).

[3] F. Aharonian et al. (HESS Collaboration), Astron. Astrophys. 457, 899 (2006) (from Fig. 13a, 20).
[4] J. Albert et al. (MAGIC Collaboration), Astrophys. J. 674, 1037 (2008).

[5] R. G. Wagner, for the VERITAS Collaboration, Proc. 31st ICRC, Lodz (2009) [arXiv:0910.4563].

[6] The CTA Consortium, arXiv:1008.3703.

[7] L. Bergström, T. Bringmann, and J. Edsjö, Phys. Rev. D 83, 045024 (2011). 
[8] L. Bergström, P. Ullio, and J. H. Buckley, Astropart. Phys. 9, 137 (1998).

[9] A. Birkedal, K. T. Matchev, M. Perelstein, and A. Spray, arXiv:hep-ph/0507194.

[10] L. Bergström, T. Bringmann, M. Eriksson, and M. Gustafsson, Phys. Rev. Lett. 94, 131301 (2005).

[11] T. Bringmann, L. Bergström, and J. Edsjö, J. High Energy Phys. 01 (2008) 049.

[12] A. A. Pullen, R. R. Chary, and M. Kamionkowski, Phys. Rev. D 76, 063006 (2007); 83, 029904(E) (2011); F. A. Aharonian et al. (HEGRA Collaboration), Astron. Astrophys. 400, 153 (2003); A. A. Abdo et al., Phys. Rev. Lett. 104, 091302 (2010); G. D. Mack, T.D. Jacques, J.F. Beacom, N.F. Bell, and H. Yüksel, Phys. Rev. D 78, 063542 (2008); G. Vertongen and C. Weniger, J. Cosmol. Astropart. Phys. 05 (2011) 027.

[13] W. A. Rolke, A. M. Lopez, and J. Conrad, Nucl. Instrum. Methods Phys. Res., Sect. A 551, 493 (2005).

[14] M. Arribas and U. Schwanke, 2008, http://www-hess .physik.hu-berlin.de/public/trabajo.pdf (Fig. 4.12).

[15] F. Aharonian et al. (HESS Collaboration), Phys. Rev. Lett. 101, 261104 (2008); A. A. Abdo et al., Phys. Rev. Lett. 102, 181101 (2009).

[16] J. R. Hoerandel, Astropart. Phys. 19, 193 (2003).

[17] D. J. Fegan, J. Phys. G 23, 1013 (1997).

[18] F. Aharonian et al., Nature (London) 439, 695 (2006).

[19] F. Aharonian et al. (HESS Collaboration), Phys. Rev. Lett. 97, 221102 (2006); 97, 249901(E) (2006).
[20] P. D. Serpico and G. Zaharijas, Astropart. Phys. 29, 380 (2008).

[21] L. Pieri, J. Lavalle, G. Bertone, and E. Branchini, Phys. Rev. D 83, 023518 (2011); R. Catena and P. Ullio, J. Cosmol. Astropart. Phys. 08 (2010) 004.

[22] P. Gondolo and J. Silk, Phys. Rev. Lett. 83, 1719 (1999).

[23] V. S. Berezinsky, A. V. Gurevich, and K. P. Zybin, Phys. Lett. B 294, 221 (1992).

[24] G. R. Blumenthal, S. M. Faber, R. Flores, and J. R. Primack, Astrophys. J. 301, 27 (1986).

[25] O. Y. Gnedin and J. R. Primack, Phys. Rev. Lett. 93, 061302 (2004).

[26] M. Gustafsson, M. Fairbairn, and J. Sommer-Larsen, Phys. Rev. D 74, 123522 (2006).

[27] J. Hisano, S. Matsumoto, M. M. Nojiri, and O. Saito, Phys. Rev. D 71, 063528 (2005).

[28] M. Gustafsson, E. Lundström, L. Bergström, and J. Edsjö, Phys. Rev. Lett. 99, 041301 (2007).

[29] Y. Mambrini, J. Cosmol. Astropart. Phys. 12 (2009) 005.

[30] G. Servant and T.M.P. Tait, Nucl. Phys. B650, 391 (2003).

[31] G. Belanger, M. Kakizaki, and A. Pukhov, arXiv:1012.2577.

[32] A. Abramowski et al., Phys. Rev. Lett. 106, 161301 (2011).

[33] T. Bringmann, F. Calore, G. Vertongen, and C. Weniger, (work in progress). 